\title{
UAV ONBOARD PHOTOGRAMMETRY AND GPS POSITIONNING FOR EARTHWORKS
}

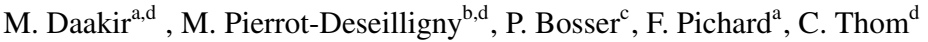 \\ ${ }^{a}$ Vinci-Construction-Terrassement, 1, Rue du docteur Charcot, 91421 Morangis, France - \\ (mehdi.daakir, francis.pichard)@vinci-construction.com \\ buniversité Paris-Est, IGN, ENSG, LOEMI , 6-8 Avenue Blaise Pascal, 77455 Champs-sur-Marne, France - \\ marc.pierrot-deseilligny@ensg.eu \\ ${ }^{c}$ ENSTA Bretagne-OSM Team, 2 rue Francois Verny, 29806 Brest, France - \\ pierre.bosser@ensta-bretagne.fr \\ ${ }^{\mathrm{d}}$ Université Paris-Est, IGN, SRIG, LOEMI, 73 avenue de Paris, 94160 Saint-Mande, France - \\ christian.thom@ign.fr
}

KEY WORDS: UAV, GPS, photogrammetry, direct georeferencing, metrology, lever-arm, delay

\begin{abstract}
:
Over the last decade, Unmanned Airbone Vehicles (UAVs) have been largely used for civil applications. Airborne photogrammetry has found place in these applications not only for 3D modeling but also as a measurement tool. Vinci-Construction-Terrassement is a private company specialized in public works sector and uses airborn photogrammetry as a mapping solution and metrology investigation tool on its sites. This technology is very efficient for the calculation of stock volumes for instance, or for time tracking of specific areas with risk of landslides. The aim of the present work is to perform a direct georeferencing of images acquired by the camera leaning on an embedded GPS receiver. UAV, GPS receiver and camera used are low-cost models and therefore data processing is adapted to this particular constraint.
\end{abstract}

\section{INTRODUCTION}

The subject of our work is at crossroads of several technological areas undergoing a complete revolution since the past 10 years: the development of light aerial vehicles like UAVs, the development of satellite positioning systems (GNSS) and the development of 3D modeling algorithmic solutions, fully automatic, based on stereoscopic images. Nowadays, by embedding a good quality commercial camera on a UAV, it is possible to achieve a visually "perfect" 3D model of a scene. In parallel, advances in GNSS receivers and positioning softwares allow high accuracy measurements and a centimeter level positionning. However, the use of these 3D models as a support of measurements for metrological purpuse can not be guaranteed because of insufficient accuracy of those models. Also, for large size projects, indirect georeferencing, based on ground control points, is limited by the number of points to be measured and the time spend on the field measuring these references. Direct georeferencing afford a considerable time saving, since at most one ground control point is necessary, and centimeter level accuracy is attended for a differential GPS processing based on carrier-phase measurements. However, to achieve a centimeter accuracy georeferencing, two important elements are to consider: the calibration of the lever arm and the time synchronization of the sensors. We compare in this article two calibration methods of the lever arm vector, the classic method called auto-calibration and a new method called pseudo-materialization. We also highlight the importance of taking into account the residual electronic delay between the sensors.

\section{HARDWARE}

Several commercial solutions emerged during the last year (2014) (e.g, senseFly eBee RTK ${ }^{1}$ and the TopCon MAVINCI Sirius Pro ${ }^{2}$ ).

\footnotetext{
${ }^{1}$ http ://www.sensefly.com

${ }^{2}$ http://www.mavinci.de/
}

The absolute accuracy announced by manufacturers for these solutions is about $3 \mathrm{~cm}$ to $5 \mathrm{~cm}$. In the academic field, studies on hybridation of GNSS positionning and photogrammetry for aerial mapping show that the state of the art is still at decimeter absolute accuracy level for low-cost solutions. The aim of our study is to achieve centimeter absolute accuracy with at most one single ground control point. This should be achievable with a relatively "low cost" system. The system will typically include:

1. a hexacopter UAV

2. a photogrammetric quality light camera (weight below $350 \mathrm{~g}$ )

3. an onboard GPS receiver synchronized with the camera

4. a fixed reference GNSS station

The GPS module chosen is the one used by the "GeoCube" (Benoi et al., 2013) a multi-sensor geo-monitoring system developed at French Mapping Agency (IGN). It is an ublox (u-blox, 2014) LEA-6T-0-001 GPS receiver. It records raw carrier-phase data on GPS constellation and on first frequency (L1) only. This allows for short baselines accurate relative positioning. Its price is relatively low (less than $100 €$ with a patch antenna (u-blox, 2009)).

The camera choosen for our experiments is the RX1 model from SONY. This compact camera is a professional full-frame $24 \mathrm{Mpx}$ sensor. The lens is a fixed focal of $35 \mathrm{~mm}$ length. The optical quality is very honorable making of the RX1 a good photogrammetric camera. However some disadvantages exist : no interchangeable optical lens capability and the weight of the camera is quite important ( $482 \mathrm{~g}$ ) which is not suitable for a small UAV. The price is also relatively high, around $2500 €$.

The UAV acquired by Vinci-Construction-Terrassement is a DJIF550 hexacopter. This UAV allows a more stabilized flight making it an exellent choice for aerial photography. One disadvantage of this model is the flying range that remains below 10 minutes. However, this UAV can take off with a payload capacity of 2 
$\mathrm{kg}$. The price is very affordable. The pre-assembled copter costs about $400 €$ online.

Figure 1 shows the prototype we achieved:

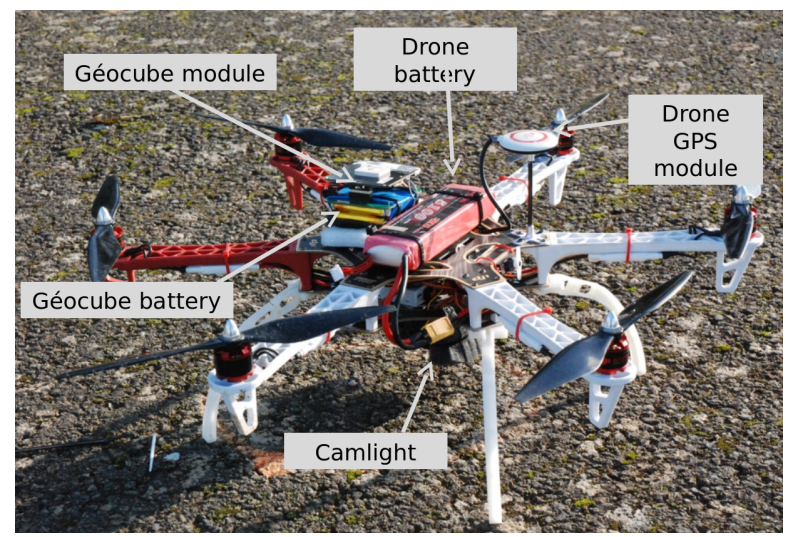

Figure 1: The drone DJI-F550 with GPS and onboard camera

\section{DATA PROCSSING AND RESULTS}

Combining photogrammetry and GPS data involves:

1. bundle block adjustement of all images

2. processing an accurate GPS trajectory

3. calibration of lever arm vector

4. time synchronization of both sensors

\subsection{Bundle Block Adjustement}

Estimation of camera poses is performed using the free open source software Apero/MicMac (Pierrot-Deseilligny and Clery, 2011). Apero uses an implementation of SIFT algorithm (Lowe, 2004) to compute tie points from pairs of images, then compute relative orientation. It is also possible to estimate intrinsec parameters of the camera by self calibration method. Several distortion models are included in Apero such as: Radial, Fraser or Fisheye. All theoretical and practical aspects concerning bundle block adjustement with Apero are discribed in MicMac official documentation (Pierrot-Deseilligny, 2015).

\subsection{GPS Processing}

As for all GNSS data processing the most important part is fixing to integers values phase ambiguities. In our case, we have a single frequency data. The computation strategy adopted is called "fixand-hold" (Benoit, 2014). The ambiguities are first fixed before UAV take-off. Next, the signal is tracked to detect possible cycle slips. With this technique the remaining unknowns to estimate are the position of the receiver at each epoch. Figure 2 shows GPS carrier-phase double difference:

The equation of double difference between 2 satellites and 2 receivers expressed in $\mathrm{ECEF}^{3}$ reference frame can be written as follow:

$$
\begin{array}{r}
\nabla \Delta\left(\Phi_{r_{i}, r_{k}}^{s_{j}, s_{l}}\right)=\nabla \Delta\left(\rho_{r_{i}, r_{k}}^{s_{j}, s_{l}}\right)-\lambda \cdot \nabla \Delta\left(N_{r_{i}, r_{k}}^{s_{j}, s_{l}}\right) \\
-\nabla \Delta\left(\tau_{r_{i}, r_{k}, \text { iono }}^{s_{j}, s_{l}}\right)-\nabla \Delta\left(\tau_{r_{i}, r_{k}, \text { tropo }}^{s_{j}, s_{l}}\right)-\nabla \Delta(\epsilon)
\end{array}
$$

${ }^{3}$ Earth-Centred Earth-Fixed (GPS uses the World Geodetic System WGS-84)

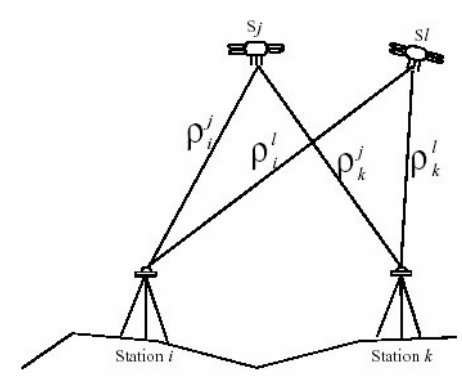

Figure 2: The double-difference geometry

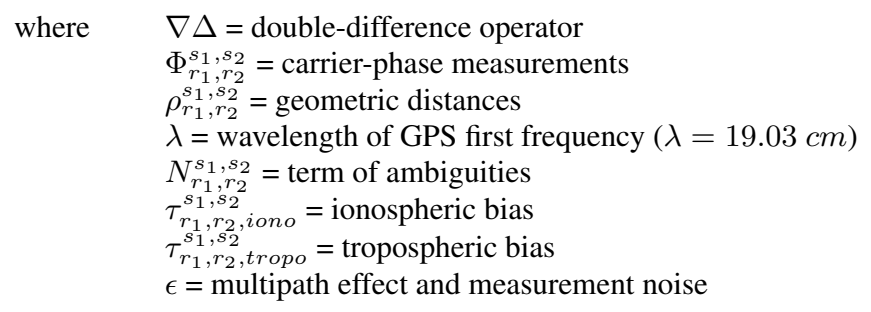

If we know at the first epoch, before UAV take-off, an approximate position of our GPS receiver with an accuracy better than $\frac{\lambda}{2} \approx 10 \mathrm{~cm}$ then the ambiguities can simply be calculated instead of being estimated using:

$$
\begin{array}{r}
N_{r_{i}, r_{k}}^{s_{j}, s_{l}}=\left\lfloor\frac { 1 } { \lambda } \cdot \left(\nabla \Delta\left(\Phi_{r_{i}, r_{k}}^{s_{j}, s_{l}}\right)-\nabla \Delta\left(\rho_{r_{i}, r_{k}}^{s_{j}, s_{l}}\right)\right.\right. \\
\left.\left.-\nabla \Delta\left(\tau_{r_{i}, r_{k}, \text { iono }}^{s_{j}, s_{l}}\right)-\nabla \Delta\left(\tau_{r_{i}, r_{k}, \text { tropo }}^{s_{j}, s_{l}}\right)\right)\right\rfloor
\end{array}
$$

where \lfloor\rfloor$=$ nearest integer operator

\subsection{Lever-arm calibration}

Camera and GPS receiver are linked with an offset called "leverarm". This vector separates the GPS antenna phase center and the camera optical center. We will present two methods to estimate this vector. A first one called aut-calibration method and requires the presence of ground control points. A second one called pseudo- materialization method that requires any ground control point.

3.3.1 Auto-calibration method One classical way to determine lever-arm vector is to record aerial data provided with ground control points and to estimate lever-arm as an unknow by minimizing the system of equations 3 for $N$ images:

$$
\forall i \in \llbracket 1, N \rrbracket, f_{i}:\left\{\begin{array}{c}
\left.\overrightarrow{\mathcal{C}_{i}}\right|_{t}-\mathcal{R}_{i} \cdot \overrightarrow{\mathcal{O}}-\left.\overrightarrow{\mathcal{G}_{i}}\right|_{t}=\overrightarrow{0} \\
\cdots \cdots
\end{array}\right.
$$

where $\left.\quad \overrightarrow{\mathcal{C}}_{i}\right|_{t}=$ image center $i$ expressed in ground frame $\mathcal{R}_{i}=$ orientation of image $i$ (from camera to ground frame) $\overrightarrow{\mathcal{O}}=$ vector of lever arm in camera frame $\left.\overrightarrow{\mathcal{G}_{i}}\right|_{t}=$ GPS position of image $i$ in ground frame

In fact, we used here ground control points to express camera center $\left.\overrightarrow{\mathcal{C}}_{i}\right|_{t}$ in ground frame. However, it is necessary to do again the calibration every time the sensors are removed from the UAV. 
3.3.2 Pseudo-materialization method We propose a method that requires no ground control points. Also, it is not necessary that the sensors have the same relative positions on the UAV after each flight. First we materialize a target network on the camera and on the box of the GPS receiver as shown on Figure 3:
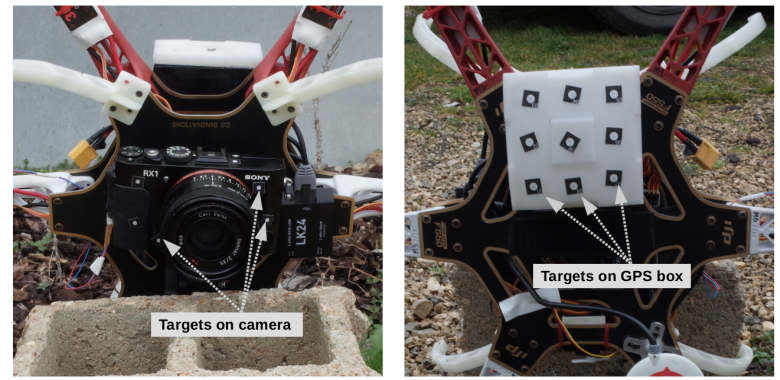

Figure 3: Targets on camera and GPS receiver box

The method consists on determining the position of the optical center of the camera and the position of the GPS antenna phase center in the local frame of each sensor (the local frame is materialized by target network)

For the camera that will be on board the UAV, we place it on a stable surface and take one image of a 3D scene. With a second camera we perform a dense acquisition around the first one. It is important during this acquisition that the camera to calibrate ${ }^{4}$ keeps the same position and orientation. Figure 4 shows the geometry of acquisition where at the center position of the camera to calibrate and around all images acquired:

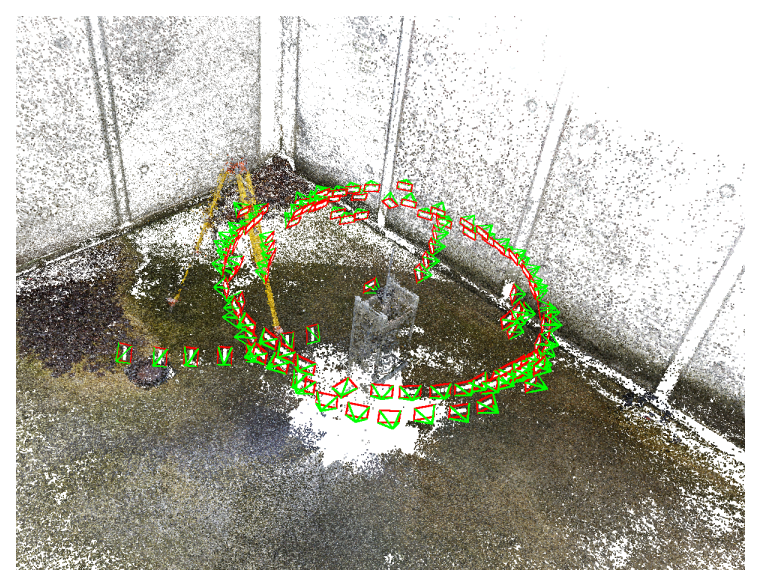

Figure 4: Geometry of acquisition for optical center calibration

Next, the model is scaled. The targets materialized on the camera are measured on the images. The 3D coordinates of each target are estimated by multi image intersection. Then, this set of coordinates are expressed in the first camera frame.

For the GPS antenna phase center, we perform four photogrammetric acquisitions of the GPS receiver. Between two acquisitions, the receiver is rotated by approximately $90^{\circ}$. Four Targets have been placed on the scene in order to convert relative orientation into absolute one. This is due to the fact that we need to express the image geometry in the same frame as the GPS receiver wich gives the position of the antenna phase center in an absolute frame. Each position of antenna phase center is processed

\footnotetext{
${ }^{4}$ We mean by calibrate to determine the relative position of the optical center in local frame. We assume that intrinsic camera parameters are known.
}

from $24 \mathrm{~h}$ carrier-phase measurements in static mode. GPS data processing was made using the free open source software RTKlib (Takasu, 2011). Figure 5 shows the acquisition images for photogrammetry processing of position taken by the GPS receiver:

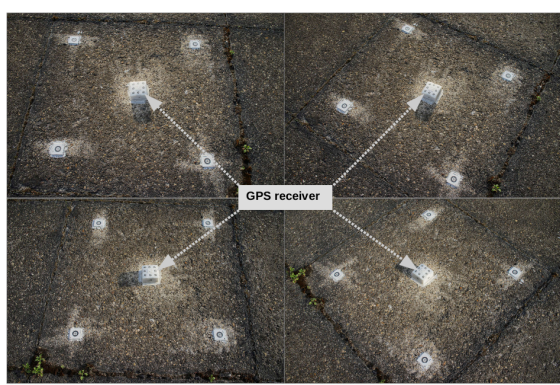

Figure 5: Images of a photogrammetric acquisition of GPS receiver

To compute lever-arm value, before UAV take off, we make a photogrammetric acquisition of the UAV to perform a 3D modeling of sensors configuration. Figure 6 reports acquisition on the left and relative orientation on the right:

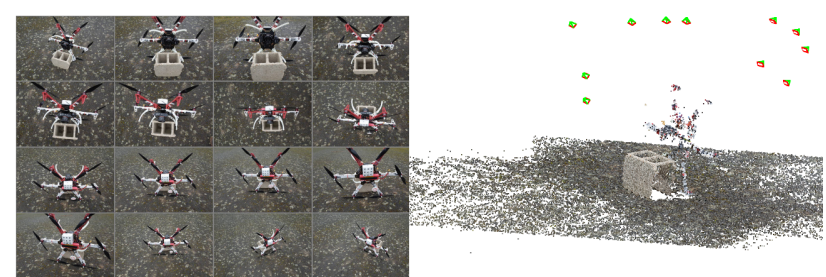

Figure 6: Modelisation of relative position of camera and GPS receiver

Lever-arm vector is computed as follow:

1. express relative orientation into camera frame system using camera targets coordinates from calibration as ground control points

2. estimate by multi image intersection GPS targets coordinates into camera frame

3. compute phase center position into camera frame by estimating 6 parameters of rigid transformation between coordinates of GPS targets into camera frame and from calibration (absolute frame)

4. lever-arm is the vector between optical center position and antenna phase center expressed in camera frame

\subsection{Time synchronisation}

Important point in the use of different data sources is the guarantee of the same timescale. Time synchronization between GPS measurements and images taken by the camera can be neglected for example if we have a dense sampling of GPS data or if the value of electronic delay is small taking into account the speed of the UAV. To achieve synchronization the GPS receiver triggers the camera. Thanks to LOEMI laboratory the SONY-RX1 camera was customized (Figure 7).

We rely on u-blox TimePulse (u-blox, 2013) functionality providing clock pulses with configurable duration. Typical duration 

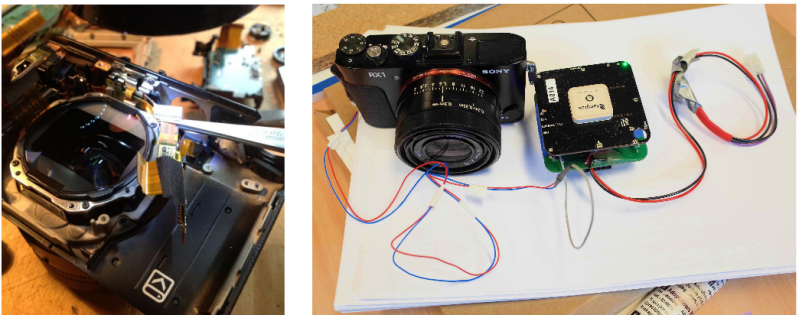

Figure 7: Adapting camera triggering with GPS module pulse $@$ LOEMI

values in our UAV configuration are 2 to 3 seconds. Also, an experimental measurement of the electronic delay was achieved at the laboratory. The measurement was carried out with an oscilloscope that shows both the waveform of the two electrical signals from the GPS pulse that triggers the camera and flash pulse which is assumed to be perfectly synchronized with the outbreak of the camera. Figure 8 reports the extent of the two signals, once for IGN LOEMI home made camera CamLight (Martin et al., 2014) and for SONY-RX1 camera. The red curve corresponds to the GPS pulse whose rising edge starts taking pictures with a delay on the falling edge of the flash pulse of $0.4 \mathrm{~ms}$ for the first camera and $64 \mathrm{~ms}$ for the second one. For SONY-RX1 camera this delay corresponds with a value of typical UAV speed of $4 \mathrm{~m} / \mathrm{s}$ to a position error of $25 \mathrm{~cm}$ (which essentially impacts the horizontal components) while for CamLight the electronical delay value allows to neglect this correction.
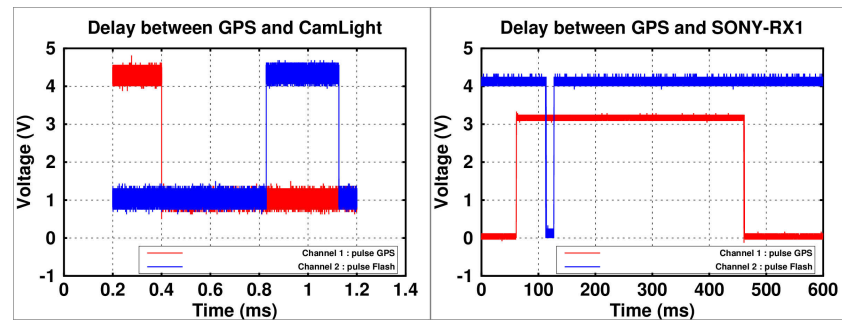

Figure 8: Measurement of electronic delay (C) O. Martin

To take into account this electronic delay, instantaneous speed of the UAV are recorded due to the GPS receiver. Hence a correction is added to system of equations 3 :

$$
\forall i \in \llbracket 1, N \rrbracket, f_{i}:\left\{\begin{array}{c}
\left.\overrightarrow{\overrightarrow{\mathcal{C}}_{i}}\right|_{t}-\mathcal{R}_{i} \cdot \overrightarrow{\mathcal{O}}-\overrightarrow{\mathcal{V}_{i}} \cdot t_{d}-\left.\overrightarrow{\mathcal{G}_{i}}\right|_{t}=\overrightarrow{0} \\
\cdots \cdots
\end{array}\right.
$$

where $\quad \overrightarrow{\mathcal{V}}_{i}=$ instantaneous speed for image $i$

$$
t_{d}=\text { time delay parameter }
$$

An experimental acquisition was performed with the UAV configuration as presented in Figure 1. Images were acquired at a rate of 3 seconds and GPS measurements are sampled at $1 \mathrm{~Hz}$ frequency (84 images for this test). Figure 9 gives a comparaison between the residuals of minimization by least square adjustement computed using system of equations 3 (in red) and equations 4 (in blue) using ground control points and relative orientation from bundle block adjustement using tie points:

Taking into account the electonic delay between the GPS receiver and the camera improve the precision by almost a factor 2 for

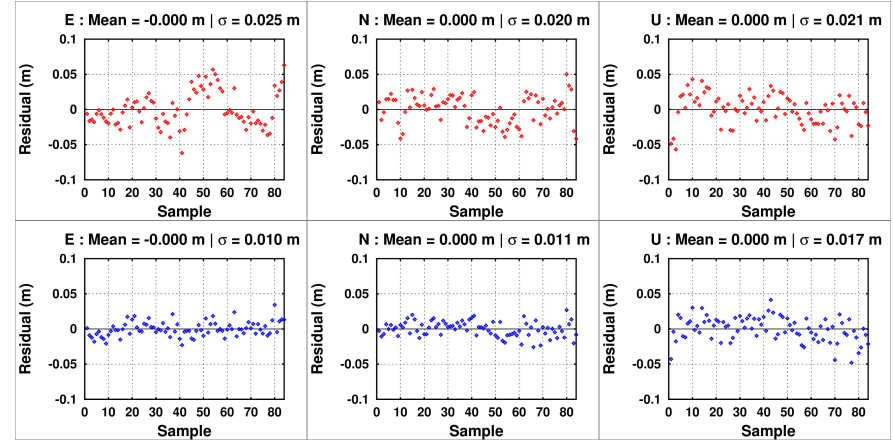

Figure 9: Comparison of residuals with and without delay

planimetric components. In fact, variations of the UAV trajectory according to the vertical component are very low. Relative accuracy obtained is at centimeter level between trajectory computed with GPS data and image trajectory computed after bundle block adjustement.

\subsection{Results}

A data acquisition was achieved composed of 134 images. For this flight we have 7 satellites above an elevation mask of $15^{\circ}$ and a $\mathrm{SNR}^{5}$ value better than $36 \mathrm{dBHz}$. Figure 10 gives residuals (eq. 5) of estimation of GPS position at each epoch for each double difference equation formed:

$$
\forall i \in \llbracket 1, P \rrbracket\left\{\begin{array}{c}
\ldots \ldots \\
\hat{V}_{i}^{m}=\nabla \Delta\left(\tilde{\Phi}_{i}^{m}\right)_{\text {mod }}-\nabla \Delta\left(\Phi_{i}^{m}\right)_{o b s} \\
\ldots \ldots
\end{array}\right.
$$

where $\quad P=$ number of double difference equations at epoch $m$ $\hat{V}_{i}^{m}=$ residual of equation $i$ at epoch $m$ $\nabla \Delta\left(\tilde{\Phi}_{i}^{m}\right)_{\text {mod }}=$ predicted carrier-phase double difference $\nabla \Delta\left(\Phi_{i}^{m}\right)_{o b s}=$ measured carrier-phase double difference

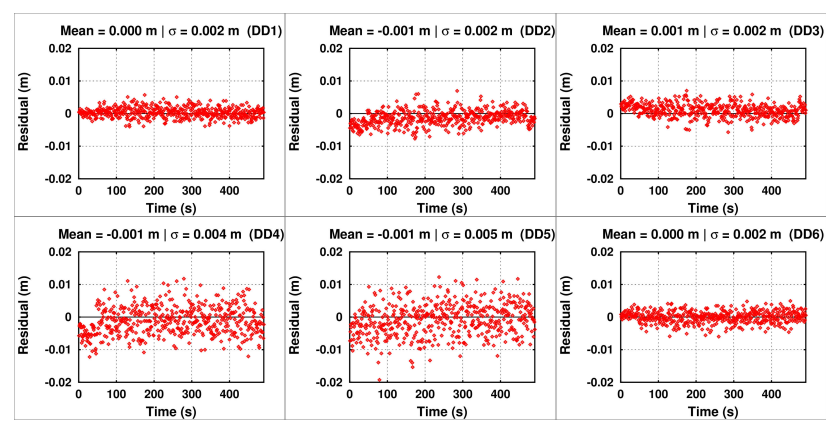

Figure 10: Residuals of GPS estimation of position at each epoch

Figure 10 highlights that model fits data well. No trend in residuals distribution is present. Amplitude of variation is at centimetric level with a constant standard deviation. Next, a measurement of lever-arm vector is performed following the method of pseudomaterialization presented in part 3.3.2. Further to the 7 parameters of the $3 \mathrm{~d}$ similarity, electronic delay is estimated while the lever arm value is fixed using system of equations 6 for $N$ images:

\footnotetext{
${ }^{5}$ Signal to Noise Ratio
} 
$\forall i \in \llbracket 1, N \rrbracket, f_{i}:\left\{\begin{array}{c}\mathcal{S}\left(\left.\overrightarrow{\mathcal{C}_{i}}\right|_{r}\right)-\mathcal{R}_{i} \cdot \overrightarrow{\mathcal{O}}-\overrightarrow{\mathcal{V}_{i}} \cdot t_{d}-\left.\overrightarrow{\mathcal{G}}_{i}\right|_{t}=\overrightarrow{0} \\ \cdots \cdots\end{array}\right.$

where $\quad \mathcal{S}\left(\left.\overrightarrow{\mathcal{C}_{i}}\right|_{r}\right)=\left.\overrightarrow{\mathcal{C}_{i}}\right|_{t}=\overrightarrow{\mathcal{T}}+\left.\mu \cdot R \cdot \overrightarrow{\mathcal{C}_{i}}\right|_{r}$ (3d similarity)

$\left.\overrightarrow{\mathcal{C}_{i}}\right|_{r}=$ image center $i$ expressed in relative frame

$\left.\overrightarrow{\mathcal{C}_{i}}\right|_{t}=$ image center $i$ expressed in ground frame

$T=$ global translation

$\mu=$ scale factor

$R=$ global rotation

Figure 11 gives residuals of system of equations 6:
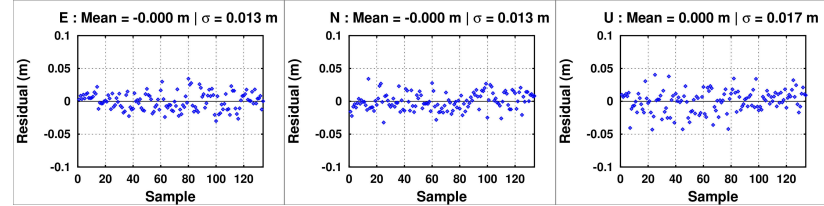

Figure 11: Residuals of 3d similarity and delay estimation

Precision on residuals after estimation of $3 \mathrm{~d}$ similarity and electronical delay is belox $2 \mathrm{~cm}$ for all axial components. The value estimated for electornic delay is $93 \mathrm{~ms}$. Residuals on check points are computed to evaluate the absolute accuracy of our prototype using pseudo-materialization method for lever-arm calibration:

\begin{tabular}{|c|c|c|c|c|}
\hline Nom Pt & $\delta x(\mathrm{~cm})$ & $\delta y(\mathrm{~cm})$ & $\delta z(\mathrm{~cm})$ & $\|\delta\|(\mathrm{cm})$ \\
\hline$P t_{1}$ & -1.5 & -0.7 & 1.0 & 2.0 \\
$P t_{2}$ & 0.7 & 1.9 & 1.8 & 2.7 \\
$P t_{3}$ & 0.3 & 1.7 & 2.3 & 2.9 \\
$P t_{4}$ & 0.6 & 1.9 & 1.8 & 2.8 \\
$P t_{5}$ & 0.4 & 1.8 & 1.2 & 2.3 \\
$P t_{6}$ & 0.4 & 3.1 & 0.8 & 3.3 \\
$P t_{7}$ & 0.3 & 3.2 & 2.2 & 3.9 \\
$P t_{8}$ & 0.8 & 2.9 & 2.4 & 3.9 \\
$P t_{9}$ & 0.5 & 2.3 & 1.1 & 2.7 \\
$P t_{10}$ & 0.0 & 2.6 & 0.4 & 2.6 \\
\hline mean & 0.2 & 2.0 & 1.5 & 2.9 \\
\hline$s t d$ & 0.6 & 1.1 & 0.6 & 0.6 \\
\hline
\end{tabular}

Table 1: Residuals on check points for pseudo-materialization method

Table 1 shows that pseudo-materialization method for lever-arm calibration can perform for this dataset a $3 \mathrm{~cm}$ accuracy on check points with a centimetric precision without using any ground control point. Next, auto lever-arm calibration method is performed using 6 ground control points. Figure 12 gives residuals of adjustement using system of equations 4 :

Precision of estimation of lever-arm using 6 ground control points is at centimetric level. Deviation is more important for vertical component. The value estimated for electornic delay is $91 \mathrm{~ms}$. Table 2 gives residuals on 4 check points after using 6 ground control points for lever arm auto-calibration:

Table 2 highlights that bias in residuals is almost 2 times less important than value of Table 1. Deviations gives an indication of the accuracy that we can achieve with our system using a few ground control points to calibrate lever-arm vector. To maintain

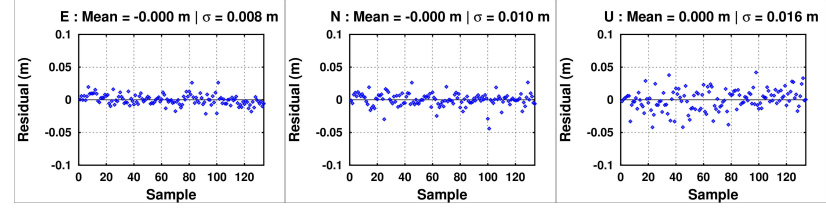

Figure 12: Residuals of lever-arm and delay estimation using ground control points

\begin{tabular}{|c|c|c|c|c|}
\hline Nom Pt & $\delta x(\mathrm{~cm})$ & $\delta y(\mathrm{~cm})$ & $\delta z(\mathrm{~cm})$ & $\|\delta\|(\mathrm{cm})$ \\
\hline$P t_{2}$ & -0.8 & -0.7 & -0.4 & 1.2 \\
$P t_{6}$ & -1.2 & 0.4 & -1.4 & 1.9 \\
$P t_{7}$ & -1.3 & 0.2 & -0.1 & 1.3 \\
$P t_{10}$ & -1.4 & 0.1 & -1.8 & 2.3 \\
\hline mean & -1.1 & 0.0 & -0.9 & 1.7 \\
\hline
\end{tabular}

Table 2: Residuals on check points for auto-calibration method

this lever-arm calibration valid for the following acquisitions, and no longer need to have ground control points, it is necessary to have a mechanically stable system to replace the sensors at the same position, and orientation for the camera, on the UAV. This problem is not yet solved for our prototype.

Auto-calibration method presents a higher accuracy because of the use of ground control points. The second method has the advantage of flexibility: no ground control point is necessary and sensors can change relative position/orientation on the UAV, only a photogrammetric acquisition is necessary before UAV take off. However, bias is more important for this method. A possible explanation for the presence of this bias is that the estimation of intrinsec parameters of the camera, especially the focal length, is not optimal after the bundle block adjustment. The presence of a high correlation between the intrinsec camera parameters and lever-arm vector makes us think that the error on the intrinsec parameters is compensated in the estimation of the lever arm with auto-calibration method, that is why bias is less important for second method, which is not the case for the first method.

\subsection{Further work}

Next experiments involve comparaison between our single-frequency prototype presented in part 2. and a dual-frequency GNSS receiver. In fact, a second prototype is under developpement combining a Hemisphere Eclipse P 303 compact OEM $^{6}$ GNSS module and a Tallysman dual-frequency GNSS antenna. A Raspberry $\mathrm{Pi}$ has been chosen to manage the GNSS receiver startup and recording raw data on a memory card. Figure 13 presents our new prototype:

Indeed, the new LOEMI home made camera CamLight is now available. The CamLight is a camera designed for aerial photography by UAV. It has characteristics that make it a high-quality photogrammetric camera (full frame sensor, fixed focal lens, no autofocus, global shutter, low mass, ...) and has a negligible electronic delay.

\section{CONCLUSIONS}

Commercial solutions that offer UAV dedicated to acquisition of aerial images based on GNSS module for direct-georeferencing still have a significant cost, around $25-50 \mathrm{k} €$ to our knowledge. Through our precedent results, we show that it is possible with a single-frequency GPS module and an acceptable photogrammetric quality camera to achieve few centimeters accuracy for short

\footnotetext{
${ }^{6}$ Original Equipment Manufacturer
} 


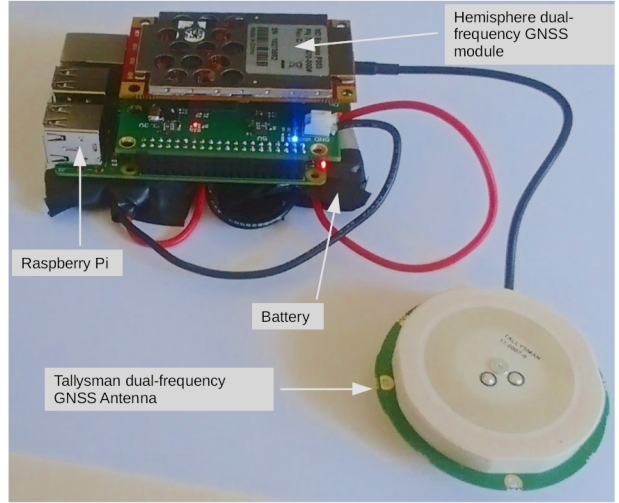

Figure 13: Dual-frequency GNSS receiver

baselines. It is possible to further enhance the accuracy of our results by combining with more constraint both techniques (the relative precision of photogrammetry and absolute precision offered by GPS). An adjustement of all sources of observations, tie points, ground control points and GPS positions, with an optimal weighting, can further improve our results.

\section{ACKNOWLEDGEMENTS}

We thank here IGN LOEMI laboratory and especially O. Martin for all his support and help in solving hardware problems. Vinci-Construction-Terrassement for its support and funding this research. Sebastien Patte for working on the development of our second prototype.

\section{REFERENCES}

Benoit, L., 2014. Positionnement GPS précis et en temps-réel dans le contexte de réseaux de capteurs sans fil type Geocube : application à des objets géophysiques de taille kilométrique. $\mathrm{PhD}$ thesis, École Normale Supérieure.

Benoit, L., Thom, C. and Martin, O., 2013. Landslide monitoring using Geocubes, a wireless network of low-cost GPS receivers. In: EGU General Assembly Conference Abstracts, EGU General Assembly Conference Abstracts, Vol. 15, p. 6995.

Bláha, M., Eisenbeiss, H., Grimm, D. and Limpach, P., 2011. Direct georeferencing of uavs. ISPRS - International Archives of the Photogrammetry, Remote Sensing and Spatial Information Sciences XXXVIII-1/C22, pp. 131-136.

Bosser, P., 2012. GNSS : Systèmes globaux de positionnement par satellite. Cours de l'École Nationale des Sciences Géographiques.

Estey, L. H. and Meertens, C. M., 1999. Teqc: the multi-purpose toolkit for gps/glonass data. GPS solutions 3(1), pp. 42-49.

Fath-Allah, T. F., 2010. A new approach for cycle slips repairing using gps single frequency data. World Applied Sciences Journal 8(3), pp. 315325 .

GNSS DATA PROCESSING Volume I: Fundamentals and Algorithms, 2013. ESA Communications.

Klobuchar, J. et al., 1987. Ionospheric time-delay algorithm for singlefrequency gps users. Aerospace and Electronic Systems, IEEE Transactions on (3), pp. 325-331.

Lowe, D. G., 2004. Distinctive image features from scale-invariant keypoints. International journal of computer vision 60(2), pp. 91-110.

Martin, O., Meynard, C., Pierrot-Deseilligny, M., Souchon, J. and Thom, C., 2014. Réalisation d'une caméra photogrammétrique ultralégère et de haute résolution. http://drone.teledetection.fr/.

Pfeifer, N., Glira, P. and Briese, C., 2012. Direct georeferencing with on board navigation components of light weight uav platforms. ISPRS - International Archives of the Photogrammetry, Remote Sensing and Spatial Information Sciences XXXIX-B7, pp. 487-492.
Pierrot-Deseilligny, M., 2015. MicMac, Apero, Pastis and Other Beverages in a Nutshell! MicMac documentation.

Pierrot-Deseilligny, M. and Clery, I., 2011. Apero, an open source bundle adjusment software for automatic calibration and orientation of set of images. ISPRS - International Archives of the Photogrammetry, Remote Sensing and Spatial Information Sciences XXXVIII-5/W16, pp. 269276.

Rehak, M., Mabillard, R. and Skaloud, J., 2013. A micro-uav with the capability of direct georeferencing. ISPRS - International Archives of the Photogrammetry, Remote Sensing and Spatial Information Sciences XL-1/W2, pp. 317-323.

Saastamoinen, J., 1972. Atmospheric correction for the troposphere and stratosphere in radio ranging satellites. The use of artificial satellites for geodesy pp. 247-251.

Stempfhuber, W. and Buchholz, M., 2011. A precise, low-cost rtk gnss system for uav applications. ISPRS - International Archives of the Photogrammetry, Remote Sensing and Spatial Information Sciences XXXVIII-1/C22, pp. 289-293.

Takasu, T., 2011. Rtklib: An open source program package for gnss positioning.

Takasu, T. and Yasuda, A., 2008. Evaluation of rtk-gps performance with low-cost single-frequency gps receivers. In: Proceedings of international symposium on GPS/GNSS, pp. 852-861.

Tournadre, V., Pierrot-Deseilligny, M. and Faure, P. H., 2014. Uav photogrammetry to monitor dykes calibration and comparison to terrestrial lidar. ISPRS - International Archives of the Photogrammetry, Remote Sensing and Spatial Information Sciences XL-3/W1, pp. 143-148.

Turner, D., Lucieer, A. and Wallace, L., 2014. Direct georeferencing of ultrahigh-resolution uav imagery. Geoscience and Remote Sensing, IEEE Transactions on 52(5), pp. 2738-2745.

u-blox, 2009. GPS Antennas RF Design Considerations for u-blox GPS Receivers. u-blox AG, www.u-blox.com.

u-blox, 2013. u-blox 6 Receiver Description Including Protocol Specification. Gps.g6-sw-10018-f edn, u-blox AG, www.u-blox.com.

u-blox, 2014. LEA-6 u-blox 6 GPS Modules Data Sheet. u-blox AG, www.u-blox.com. Objective Specification. 